\title{
Could Mobile Phone Electromagnetic Radiation Affect Thyroid Function?
}

\section{Tsatsaris Athanasios*}

Sanitary Police Colonel Department of Grevena,

University of Athens, Greece

*Corresponding author: Tsatsaris Athanasios,

Sanitary Police Colonel Department of Grevena,

University of Athens, 4 Ag. Kosma Street, 51100, Grevena,

Greece

Received: J une 28, 2018; Accepted: J une 29, 2018;

Published: J uly 06, 2018

\section{Editorial}

The last decade the number of mobile phones used worldwide has reached extraordinary level. It is well known, that electromagnetic radiation emitted by various devices under certain circumstances, may produce biological effect on humans. Is Cell phone an exception? Scientific research has started to focus on this new challenge. Could mobile phone Electromagnetic Radiation (ER) affect Thyroid function?.

Thyroid function is mostly based upon Thyroid Gland (TG). TG is located anteriorly in the lower neck, covering the distance from the fifth cervical vertebra till to the first thoracic. Is highly vascularised and innervated by the autonomic nervous system. The produced hormones are Triiodothyronine (T3) and Thyroxine (T4), under regulation of Thyroid Stimulating Hormone (TSH) which is produced in pituitary gland and regulated in turn by Thyrotropin Releasing Hormone (TRH), finally produced in Hypothalamus. Moreover, Thyroid gland secrets the hormone calcitonine which is implicated in homeostasis of blood calcium level. Thus, the distance between TG-pituitary gland-hypothalamus and mobile phone while on operation, is approximately $15-45 \mathrm{~cm}$. Within this distance, ER seems to be capable of inducing biochemical phenomena on Thyroid operational axis.

Thyroid gland plays a predominant role in Human organism. Not only serves as an outstanding regulator of metabolism but also affects in many different ways almost all the other systems. It has the ability to accelerate or decelerate all biochemical reactions inside and outside cell environment.
Biological systems such as Cardiovascular, pulmonary, gastrointestinal and nervous system are mostly related with thyroid hormones, through simple or complicated feed-back algorithms that are still being under research worldwide. So, it is easily deduced that possible effect of ER on Thyroid operational axis might also deranges the proper function of the aforementioned systems.

So far, ER is considered to produce non thermal intervention on TG function. Specifically, Radiofrequency spectrum is emitted by the cell phones and the closest the antenna to the body tissues the higher the absorption rate. The emitted radiation is measured by the Specific Absorption Rate (SAR). Interestingly, different cell phone models exhibit different SARs. For protection reasons, upper SAR limits have been determined and followed by companies worldwide. One characteristic upper limit is the $1,6-2 \mathrm{Watt} / \mathrm{Kg}$ for most countries.

ER actually represents electromagnetic waves of a specific frequency that might cause biochemical effects on TG-axis. Also, Electromagnetic Waves possess certain attributes derived by their nature. The incident wave towards a surface propagates, retransmitted or reflected. Choosing the appropriate material the course of the wave may be predicted and modified. Thus, neck human area may be covered with a specific complex dielectric material with low refractive index and high reflective behavior. In this way it may be possible to avoid or minimize the ER absorption and eventually to preserve Thyroid function as much as possible.

In conclusion, the first studies on this issue have revealed that TSH values seem to have risen after extensive use of mobile phones. These preliminary results deserve further scientific research because cell phones have conquered and dominate every part of human life. 